\title{
Addiction Treatment Aftercare Outcome Study
}

\author{
Akikur Mohammad, Kristopher J. Irizarry, Rebecca Ninah Shub, Alexandria Sarkar \\ Department of Psychiatry, LAC-USC, Los Angeles, CA, USA \\ Email: rebeccaninah@gmail.com
}

How to cite this paper: Mohammad, A., Irizarry, K.J., Shub, R.N. and Sarkar, A. (2017) Addiction Treatment Aftercare Outcome Study. Open Journal of Psychiatry, 7, 51-60.

http://dx.doi.org/10.4236/ojpsych.2017.71005

Received: November 12, 2016

Accepted: January 8, 2017

Published: January 11, 2017

Copyright $\odot 2017$ by authors and Scientific Research Publishing Inc. This work is licensed under the Creative Commons Attribution International License (CC BY 4.0). http://creativecommons.org/licenses/by/4.0/

\begin{abstract}
Aftercare is crucial once an individual has completed drug or alcohol treatment and is in recovery. There is a continuity of care that should be followed once initial treatment is completed. This usually involves a lower level of treatment such as outpatient care and a sober living environment. In order to assess the efficacy and benefit of our addiction treatment program, we investigate a set of patients in which addiction treatment outcome and rehabilitation is determined for patients who have completed treatment and followed up. We determine abstinence rates and identify predictors of treatment outcome.
\end{abstract}

\section{Keywords}

Addiction, Treatment, Dual Diagnosis, Dependence, Abstinence, Naltrexone, Vivitrol, Antabuse, Acamprosate

\section{Introduction}

Addiction is a serious problem affecting between 20 million and 40 million individuals in the United States. The economic impact on the country is estimated to be $\$ 200$ billion dollars per year in terms of lost productivity, health-related treatment costs, and criminal justice expenses [1]. The Minnesota Model of addiction treatment utilizes the Twelve-Step Facilitation program from Alcoholics Anonymous. This approach began at the state hospital in Willmar, Minnesota in 1950 and spread to the Hazelden Foundation and eventually throughout the country [2]. Hazelden's model has been assessed to determine the outcome for patients. An analysis of 1083 male and female patients identified a 1-year abstinence rate of 53\% [3].

A recent study investigating the outcome for 284 patients receiving treatment for addiction in outpatient treatment, residential treatment and sober living environments identified 1-year abstinence rates of $16.8 \%, 11.7 \%$, and $23.8 \%$ respectively. When considering across all three treatments, 1 -month abstinence rate was $74.6 \%$; 3 -month abstinence was $63.7 \%$; 6-month abstinence was $55.7 \%$; and 1 -year abstinence rate was $42.1 \%$ [4]. In a separate study, the efficacy associated with self-help groups and psy- 
chotherapy was explored in a group of 200 outpatients (28.5\% attended self-help groups and $14.5 \%$ participated in group therapy). The results indicated that 1-month abstinence was $19.3 \%$; 6-month abstinence was $22.8 \%$; and 1-year or longer abstinence was $43.9 \%[5]$.

Similar rates of abstinence have been reported for substance specific treatment programs. For example, in a study of 202 outpatients treated for tobacco-dependence, $43 \%$ abstained for at least 7 days after completing the treatment program [6]. Some studies have sought to investigate demographic features that may be associated with treatment success. In one study, African American and Latino smokers of menthol cigarettes exhibited lower 1-month abstinence (30\% and $23 \%$, respectively) when compared to white smokers (43\%) of menthol cigarettes [7].

Efforts aimed at developing successful treatment programs for addiction have led to the exploration of invasive brain based methods of treatment. In a study of five patients undergoing deep brain stimulation of the nucleus accumbens for the treatment of alcohol dependence, $40 \%$ exhibited long-term abstinence [8]. A treatment method designed to accomplish surgical ablation of the nucleus accumbens was performed in 1167 patients in China. Within a set of 272 patients undergoing this invasive surgical treatment, 5-year abstinence was documented as 58\%. In another group of 150 cases, the non-relapse rate was reported as 50\% [9].

In order to assess the efficacy and benefit our addiction treatment program, we investigate a set of patients in which addiction treatment outcome and rehabilitation is determined for patients who have completed treatment and followed up. We determine abstinence rates and identify predictors of treatment outcome.

\section{Methods}

\subsection{Detoxification}

Patients are assessed for treatment based on a dual diagnosis consisting of substance abuse coupled with mental health co-morbidity. Upon admission into the program patients undergo detoxification. Nurses acquire relevant intake history including what substance is used, how much of the substance is used, duration of use for each substance and frequency. That information is used to customize a patient specific detoxification protocol, conditions and length. Detox for each patient is individualized by MD's who are board certified in both addiction medicine and psychiatry. The resulting individualized detox tapers utilize four FDA approved addiction drugs: naltrexone, antabuse, acamprosate, and vivotrol. Taper duration ranges from 1 - 2 weeks and may be extended if necessary.

\subsection{Treatment}

Duration of treatment is optimized for each patient based a variety of factors including types of substances abused, and frequency/duration of abuse history. Typical treatment durations last 30 - 45 days and may extend as long as 90 days. Treatment consists of various modalities of science and evidence based therapies. Patients attend group therapy 3 - 4 times daily and individual sessions 4 - 5 times per week. 
Pharmacological therapies used during treatment include Naltrexone which is FDA approved for treating alcoholism and administered orally. Vivitrol is a longer acting opioid antagonist given via injection once a month. Antabuse is an acetaldehyde dehydrogenase inhibitor used to reduce craving and acamprosate stabilizes glutamate and GABA neurotransmitters during alcohol withdrawal. Additional medications may be included in treatment and thereafter, provided they have been proven to be effective in addiction clinical studies.

\subsection{Aftercare}

A component of the treatment program includes facilitating an effective aftercare plan for each patient. This is accomplished using teams of doctors and treatment program support staff in structured interactions with patients. The goal of an aftercare plan is to seamlessly transition a patient into an independent substance free lifestyle. Aftercare is typically successful when a patient fully participates in the plan-facilitation process. Completing a drug/alcohol treatment program is a feat but the battle is still being fought which is why aftercare is important in maintaining sobriety.

\subsection{Aftercare Follow Up}

Once patients exit the program, a follow up plan is enacted lasting up to a year. During this time patients are contacted by phone and asked questions about substance use, treatment efficacy and adherence to the aftercare plan. Responses to phone calls are recorded in patient records.

\subsection{Data Analysis}

Seventy-two patients were included in this study. Demographic data for patients was collected and analyzed to determine average age for all patients and for males and females. This data was used to characterize treatment outcome by age and gender over 12-month post-treatment period. Treatment outcome was analyzed to identify any relationship between treatment length and treatment efficacy.

In order to identify predictors of addiction relapse, three different levels of treatment success based on stringency of aftercare follow up data. Three different definitions for treatment success were defined and the treatment outcome data was analyzed under each of the different stringencies. The results of this analysis were used to identify predictors of addiction relapse following treatment.

\section{Results}

\subsection{Patient 12-Month Treatment Efficacy by Age and Gender}

A total of 72 patients underwent clinical treatment for addiction for which 32 were male and 40 were female. The average age of all patients was 30.36 years (s.d. $=11.3$ years) with very similar ages between the genders. The average age of males was 30.0 years ( $\mathrm{s} . \mathrm{d}=10.4 \mathrm{yrs}$ ) and the average age of females was 30.7 years (s.d. $=12.2 \mathrm{yrs}$ ). Overall, the average age of patients who successfully completed the treatment was 29.2 years (s.d. $=11.6 \mathrm{yrs}$ ) while the average age of those who failed to complete treatment 
successfully was 29.0 years (s.d. $=10.6$ yrs).

The average age of male patients who successfully completed treatment was 26.7 years (s.d. $=8.6 \mathrm{yrs}$ ) while the average age of female patients who successfully completed the treatment was 31.8 years (s.d. $=12.9 \mathrm{yrs}$ ). Interestingly, the average age of men who did not successfully complete treatment was 35.6 years (s.d. $=10.7$ yrs) while the average age of women who failed to complete treatment successfully was 28.8 years (s.d. $=10.5$ yrs).

\subsection{Statistical Analysis of Patient Demographic Factors Associated with Treatment Efficacy}

We chose to investigate if patient gender or age was a factor associated with treatment outcome. In our study, a total of 72 patients underwent clinical treatment for addiction for which 32 were male and 40 were female. The average age of males was 30.0 years $(\mathrm{s} . \mathrm{d}=10.4 \mathrm{yrs})$ and the average age of females was 30.7 years $(\mathrm{s} . \mathrm{d} .=12.2 \mathrm{yrs})$. There was no statistically significant difference between the average age of males and the age of females in our study population ( $\mathrm{p}$-value $=0.3966$, alpha $=0.05$ ).

The average age of all patients who successfully completed the treatment was 29.2 years (male and female, $\mathrm{n}=45$, s.d. $=11.6 \mathrm{yrs}$ ) while the average age of all those who failed to complete treatment successfully was 29.0 years (male and female, $\mathrm{n}=27$, s.d. $=$ $10.6 \mathrm{yrs})$. There was no statistically significant difference between the average ages of patients who successfully completed treatment versus those for which treatment was not successful $(\mathrm{p}=0.52972$, alpha $=0.05)$.

As no significant difference was identified between the average age of patients by gender, or by treatment outcome, we next wondered if there was a significant difference between the average age of males patients who successfully completed treatment $(\mathrm{n}=$ 23 , avg $=26.7 \mathrm{yrs}$, s.d. $=8.6 \mathrm{yrs})$ versus the average age of female patients who successfully completed the treatment $(\mathrm{n}=22,31.8 \mathrm{yrs}$, s.d. $=12.9 \mathrm{yrs})$. Again, although the p-value was just barely larger than 0.05 , there was no statistically significant difference between the average age of successfully treated men versus women $(p=0.06551$, alpha $=0.05$ ). In a similar manner, we tested to see if there was a significant difference between the average age of male patients $(\mathrm{n}=32-23=9$, avg $=35.6 \mathrm{yrs}$, s.d. $=10.7 \mathrm{yrs})$ versus female patients $(\mathrm{n}=40-22=18$, avg $=28.8 \mathrm{yrs}$, s.d. $=10.5 \mathrm{yrs})$ for whom treatment was not successful. Likewise, there was no statistically significant difference detected $(0.93256$, alpha $=0.05)$.

Overall we found no significant differences between the patient demographic factors of gender and age with 12-month treatment outcome.

\subsection{Patient 12-Month Treatment Efficacy by Treatment Length}

Of the 72 patients who participated in the treatment program, 53 had a treatment length of 30 days while just 19 underwent treatment for more than 30 days. Patients participating in the longer treatment program included those in a 33-day program ( $\mathrm{n}=$ 1), 35-day program $(\mathrm{n}=3), 40$-day program $(\mathrm{n}=2), 45$-day program $(\mathrm{n}=9)$ and a 60 -day program $(n=4)$. Patients undergoing the 30 day treatment program exhibited a $54.7 \%$ treatment success rate. In contrast, patients that participated in a treatment pro- 
gram lasting more than 30 days experienced a success rate of $84.2 \%$.

\subsection{Statistical Analysis of Clinical Treatment Length with 12-Month Treatment Efficacy}

We next wanted to investigate whether the length of the treatment program (30 days vs. more than 30 days) was significantly associated with treatment efficacy. Our initial analysis revealed that of the 53 patients undergoing the 30-day treatment program, 29 patients $(54.7 \%)$ exhibited a successful 12-month treatment outcome. In contrast, among the 19 patients that participated in a treatment program lasting more than 30 days, 16 patients (84.2\%) experienced a successful 12-month treatment outcome. To assess whether the treatment efficacy was significantly different between treatment length, we performed a two-sided Z-Test for population proportions using an alpha = 0.05 , which resulted in a p-value of 0.0226 . This result indicates that within our clinical addiction treatment program, treatment lengths greater than 30 days were significantly associated with better treatment outcome.

\subsection{Patient 12-Month Treatment Efficacy by Addiction Type}

Patients were treated for a number of chemical dependencies including alcohol, amphetamine, benzodiazepines, and opioids. Among the 72 total patients, 29 were treated for alcohol dependency (13 males, 16 females), 2 were treated for amphetamine dependency ( 2 males, 0 females), 13 were treated for benzodiazepine addiction ( 5 males, 8 females) and 28 were treated for opioid dependency ( 12 males, 16 females). The overall 12 -month treatment success rate across all patients was 62.5 percent. Overall, treatment success varied from a high of $100 \%$ for amphetamine addiction, to $69.2 \%$ for benzodiazepine dependency, with $64.3 \%$ percent for opioid dependency, and the lowest treatment success rate of $55.2 \%$ percent for alcohol addiction.

\subsection{Statistical Analysis of Patient Addiction Type with 12-Month Treatment Efficacy}

Within our after-care study, the number of patients being treated for alcohol dependency $\left(\mathrm{n} \_\right.$TXsuccess $=16, n_{-}$total $=29$ ) and opioid dependency $\left(n_{-}\right.$TXsuccess $=18$, $\mathrm{n} \_$total $\left.=28\right)$ was nearly identical. The remaining patients $\left(\mathrm{n} \_\right.$TXsuccess $=11, \mathrm{n} \_$total $=$ 15) represent 13 with benzodiazepine addiction and just 2 with amphetamine addiction. We first decided to perform statistical analysis to compare treatment efficacy between alcohol addiction and opioid addiction. Using the two-sided Z-Test for population proportions with an alpha $=0.05$, produced a p-value of 0.48392 . This result indicates that, within our clinical addiction treatment program, there was no statistically significant difference between 12-month treatment efficacies for patients treated for alcohol dependency versus patients treated for opioid dependency.

Next we tested to see if there was a significant difference between the 12-month treatment efficacy between the remaining patients (amphetamine and benzodiazepine dependencies) compared to those with alcohol dependency. The two-sided Z-Test for population proportions with an alpha $=0.05$ produced a $\mathrm{p}$-value of 0.242 indicating that there is no difference between treatment outcome between patients with alcohol 
dependency compared to those with amphetamine or benzodiazepine dependency.

Similarly, we tested to see if there was a significant difference in 12-month treatment efficacy between the amphetamine/benzodiazepine dependent patients compared to the opioid dependent patients. Once again, we employed the two-sided Z-Test for population proportions with an alpha $=0.05$ and obtained a p-value of 0.5485 , indicating that there is not a significant difference between treatment outcome for patients treated for opioid dependency versus patients treated for amphetamine/benzodiazepine dependency. No significant difference between patient addiction type and 12-month treatment outcome was identified.

\subsection{Analysis of Treatment Success Using Three Distinct Stringency Models for Efficacy}

Since our study design employed self-reporting from patients who suffered from addiction, we hypothesized that it was likely that a patient might misrepresent abstinence during the 12-month after-care follow up period. As we reviewed the phone call responses from the patients, it became clear that some patients were readily available for our phone calls, while other patients consistently failed to answer the phone. Initially, we struggled with how to best to identify legitimate reasons for missing a follow-up phone call, versus intentional avoidance associated with substance abuse relapse. Although there is no perfect strategy for accurately classifying missed phone calls, we decided to perform three separate analyses of the data using a sliding scale of stringency for assessing treatment success. Specifically, we assessed treatment efficacy at 1-month, 3-month, 6-month and 12-month time points for each of our levels of stringency based explicitly on how we interpreted unanswered phone calls.

The most stringent level of treatment success was designed to assess the lowest possible success rate, under the most rigorous criteria for considering treatment successful. In this model, only patients who answered the phone and explicitly stated that they remained substance free ("Y") for entire post-treatment interval were classified as successfully treated at each of the four time points. Under this most stringent model, $63.8 \%$ of patients exhibited success at the 1 month time point. While only $51.4 \%$ were successful at 3 months, with $38.9 \%$ successful at 6 months and just $23.6 \%$ remaining substance free 12 months after completing the treatment program.

For the moderate stringency model, we relaxed the inclusion criteria classifying patients as successful with the following three conditions for classifying a patient as successfully treated: 1) no "N" responses at all; 2) no more than 4 "NA" responses in any 12-month period; and 3) no "NA" responses allowed if they were immediately followed by a " $\mathrm{N}$ " response in the very next phone call. Under these criteria, $81.9 \%$ of patients were successful at 1 -month, $72.2 \%$ were successful at 3 -month, $62.5 \%$ were successful at 6-month and $48.6 \%$ reported success at the 12 -month time point.

Finally, in the least stringent model for treatment success, our only exclusion criteria for success was any patient that replied " $N$ " to the question of being substance free within the time period being assessed. Under this model, $95.8 \%$ of patients were successful at the 1 -month time point, $86.1 \%$ were successful at the 3 -month time point, $83.3 \%$ were successful at the 6 -month time point and $69.4 \%$ were successfully substance 
free 12-month following treatment.

\subsection{Patterns of Post-Treatment Patient Behavior with 12-Month Treatment Efficacy}

In the course of this study we analyzed 3240 clinical data points $(72$ patients $\times 15$ time points $\times 3$ stringency models) in an attempt to assess the efficacy of our addiction treatment program. Interestingly, the process of defining the inclusion and exclusion criteria for treatment success across the different stringency models offered a unique opportunity to characterize trends in patient post-treatment behavior with 12-month treatment outcome. We reasoned that certain behavioral patterns in the after-care follow up phase of our study might have prognostic value for inferring the likelihood of treatment success or failure. Ultimately, we are interested in identifying any factors associated with treatment outcome that can prove informative in identifying patients at risk for poor treatment response.

We chose to base our analysis of patient behavioral patterns using only the data contained in the most stringent model for assessing treatment outcome; in which only patients who always answered the phone and explicitly stated that they remained substance free ("Y") for the entire duration of the post-treatment interval (1-month, 3-month, 6-month \& 12-month) were classified as successfully treated. We reasoned that markers of poor treatment outcome are more easily identifiable when the classification of poor patient response is as broad and sensitive as possible.

In order to identify putative patterns of patient behavior during the after-care portion of the study, we counted the total number of "no telephone answers" (NA) and "negative responses to substance free question" $(\mathrm{N})$ for each of the 72 patients. The number of "NA's" ranged from 0 (18 patients), to 15 (1 patient). Similarly, the number of " $\mathrm{N}$ " responses ranged from 0 (47 patients) to 4 (4 patients). The average number of "NA's" per patient was 3.55 with a standard deviation of 3.92, while the average number of " $N$ " responses per patient was 0.681 with a standard deviation of 1.165. Based on the relationship between "NA" and "N" across the patients, we decided to investigate the feasibility of using the frequency of "NA's" among patients as a prognostic marker for "N" responses.

To quantify the value of specific frequencies of "NA's" as predictors of treatment outcome, we calculated the sensitivity and specificity for specific frequencies of NA among patients. Within our data set, all patients with 0 NA's also had 0 “N” responses. We next considered patients for which at least 1 NA occurred during the 12 month post-treatment period. The sensitivity for NA $\geq 1$ was 1.00 while the specificity was 0.383 . Similarly, the sensitivity associated with NA $\geq 2$ was also 1.00 while the specificity increased to 0.574 . When we calculated the sensitivity and specificity calculations for $\mathrm{NA} \geq 3$, we obtained a sensitivity of 0.92 and a specificity of 0.894 . Finally, we calculated a specificity of 0.8 and a sensitivity of 0.936 for NA $\geq 4$. During the analysis we noticed that increasing the frequency of NA's resulted in fewer true positives and more false negatives. Subsequently we selected NA $\geq 3$ as the optimal balance between sensitivity and specificity because when $\mathrm{NA} \geq 3$, true positives were 23 and true negatives were 42 while there were only 3 false positives and 5 false negatives. 


\subsection{Relative Risk of Addiction Relapse for Patients with Specific Post-Treatment Behavior}

Given the results of sensitivity and specificity calculations for different frequencies of "NA" as behavioral markers for treatment response, we identified a value of NA $\geq 3$ as exhibiting an optimal balance of high sensitivity and specificity. Subsequently, we were curious to discover if patients having at least 3 occurrences of NA during the 12-month post treatment period were at an increased relative risk for substance abuse relapse compared to patients having fewer than 3 NA's in the same period.

A total of 28 patients exhibited at least three instances of NA in the 12-month post treatment period. Of these, 23 patients exhibited evidence of unsuccessful treatment response resulting in an incidence of $23 / 28=0.8214$. In contrast, 44 patients exhibited fewer than $3 \mathrm{NA}$ instances during the 12 month post treatment period. Among these, just 2 patients experienced poor treatment outcome, associated with an incidence of 0.04545 . We calculated the relative risk of poor treatment outcome by dividing the incidence of poor treatment outcome among patients with NA $\geq 3(0.8214)$ by the incidence of poor treatment outcome among patients for whom NA $<3(0.04545)$.

The corresponding relative risk of substance abuse relapse following treatment is 18.1 for patients who fail to answer the phone at least three times during the follow up period compared to patients who only failed to answer the phone either 0,1 or 2 times during the entire 12-month post treatment period. These results strongly suggest that behavioral patterns of patients associated with non-answered phone calls exhibit a much greater relative risk of substance abuse relapse.

\section{Discussion}

This study investigated the efficacy and benefit of our addiction treatment program on patients who completed treatment and participated in aftercare follow-up. Specifically we conducted an aftercare follow study to determine abstinence rates and identify predictors of treatment outcome. Our results demonstrated that patients undergoing the 30 days treatment program exhibited a $54.7 \%$ treatment success rate while patients treated for more than 30 days experienced a success rate of $84.2 \%$ ( $p$-value $=0.0226$ ). Our results indicated no statistically significant difference in treatment outcome across addiction types of amphetamine addiction, benzodiazepine dependency, opioid dependency, and alcohol addiction.

As part of the analysis, we developed three stringency models of treatment outcome success. The low-stringency model provided the greatest treatment success rate by excluding the fewest patients from the "success" category. Low stringency model produced treatment success rates of $95.8 \%$ ( 1 month), $86.1 \%$ (3 months), $83.3 \%$ (6 months), and $69.4 \%$ (12 months) following treatment.

The moderate stringency model was constructed as an intermediate model of treatment success. Treatment outcome results were $81.9 \%, 72.2 \%, 62.5 \%$, and $48.6 \%$ for the 1 month, 3 months, 6 months and 12 months aftercare time points respectively.

The high stringency model produced the lowest possible success rate using the most rigorous criteria for considering treatment success. Under the high stringency model 
$63.8 \%$ of patients exhibited success at the 1 month time point; $51.4 \%$ were successful at 3 months; $38.9 \%$ were successful at 6 months and $23.6 \%$ remained substance free 12 months after completing the treatment program.

The rationale for choosing multiple levels of stringency in this study was to accurately and objectively assess the efficacy of our addiction treatment program. The criteria for characterizing success can sometimes be subjective based on the inclusion criteria chosen. It is common for studies to report the treatment outcome in the most favorable manner. We believe that such reporting makes it difficult to compare studies across treatment programs. Accordingly, we created multiple definitions of treatment success and assessed our data under each stringency model. Regardless of the stringency level selected, we identified a trend of higher treatment at earlier time points ( 1 month and 3 months) versus later time points (6 months and 12 months).

This study was data intensive as 3240 clinical data points $(72$ patients $\times 15$ time points $\times 3$ stringency models) were analyzed in an attempt to assess the efficacy of our addiction treatment program. In the course of this comprehensive analysis, we were able to identify trends in patient post-treatment behavior with 12-month treatment outcome. Specifically we counted the total number of "no telephone answers" (NA) and "negative responses to substance free question" (N) for each of the 72 patients.

We chose to base our analysis of patient behavioral patterns using only the data contained in the most stringent model for assessing treatment outcome based on the rationale that poor treatment outcome is most easily identified when the classification of poor patient response is as broad and sensitive as possible. Subsequently only patients who always answered the phone and explicitly stated that they remained substance free ("Y") for the entire duration of the post-treatment interval ( 1 month, 3 months, 6 months \& 12 months) were classified as successfully treated.

In order to determine if there was any specific behavioral pattern of post-treatment follow-up that might be indicative of poor treatment outcome, we selected NA $\geq 3$ as an aftercare response pattern that provided the optimal balance between sensitivity and specificity because when $\mathrm{NA} \geq 3$, treatment outcome true positives and true negatives were maximized while false positives and false negatives were minimized.

The corresponding relative risk of substance abuse relapse following treatment is 18.1 for patients who fail to answer the phone at least three times during the follow-up period compared to patients who only failed to answer the phone either 0,1 or 2 times during the entire 12-month post treatment period. These results suggest that aftercare follow-up can identify patients at risk for relapse or additional treatment.

Recovery is an ongoing process once a client leaves treatment. Clients who adhere to their discharge plan and immerse themselves in recovery related activities and lifestyle are likely to achieve sobriety for longer periods of time if not indefinitely. Clients who remain in treatment longer have higher success rates. Most clients ideally just want to return to normality once they've left inpatient treatment. At Inspire Malibu drug and alcohol treatment center, we offer aftercare follow-up as a courtesy to our clients once they leave treatment. While our clients are in treatment, we develop a rapport that allows us to maintain an aftercare relationship where we document their progress and sobriety. 


\section{References}

[1] Kuehn, B.M. (2013) Addiction: White House Seeks "Third Way": Policy Emphasizes Prevention, Treatment, Recovery. JAMA, 309, 2201-2202.

https://doi.org/10.1001/jama.2013.6001

[2] Anderson, D.J., McGovern, J.P. and Du Pont, R.L. (1999) The Origins of the Minnesota Model of Addiction Treatment-A First Person Account. Journal of Addictive Diseases, 18, 107-114. https://doi.org/10.1300/J069v18n01_10

[3] Stinchfield, R. and Owen, P. (1998) Hazelden's Model of Treatment and Its Outcome. Addictive Behaviors, 23, 669-683. https://doi.org/10.1016/S0306-4603(98)00015-X

[4] Bergman, B.G., Hoeppner, B.B., Nelson, L.M., Slaymaker, V. and Kelly, J.F. (2015) The Effects of Continuing Care on Emerging Adult Outcomes Following Residential Addiction Treatment. Drug and Alcohol Dependence, 153, 207-214. https://doi.org/10.1016/j.drugalcdep.2015.05.017

[5] Sudraba, V., Millere, I. and Rancans, E. (2012) Effectiveness of Self-Help Groups and Psychotherapy: Self-Assessment of Patients with Substance Use Disorders. Medicina (Kaunas), 48, 544-551.

[6] Khara, M. and Okoli, C.T. (2011) The Tobacco-Dependence Clinic: Intensive TobaccoDependence Treatment in an Addiction Services Outpatient Setting. The American Journal on Addictions, 20, 45-55. https://doi.org/10.1111/j.1521-0391.2010.00096.x

[7] Gandhi, K.K., Foulds, J., Steinberg, M.B., Lu, S.E. and Williams, J.M. (2009) Lower Quit Rates among African American and Latino Menthol Cigarette Smokers at a Tobacco Treatment Clinic. International Journal of Clinical Practice, 63, 360-367. https://doi.org/10.1111/j.1742-1241.2008.01969.x

[8] Voges, J., Müller, U., Bogerts, B., Münte, T. and Heinze, H.J. (2013) Deep Brain Stimulation Surgery for Alcohol Addiction. World Neurosurgery, 80, S28.e21-S28.e31.

[9] Li, N., Wang, J., Wang, X.L., Chang, C.W., Ge, S.N., Gao, L., Wu, H.M., Zhao, H.K., Geng, N. and Gao, G.D. (2013) Nucleus Accumbens Surgery for Addiction. World Neurosurgery, 80, S28.e9-S28.e19.

Scientific Research Publishing

Submit or recommend next manuscript to SCIRP and we will provide best service for you:

Accepting pre-submission inquiries through Email, Facebook, LinkedIn, Twitter, etc. A wide selection of journals (inclusive of 9 subjects, more than 200 journals)

Providing 24-hour high-quality service

User-friendly online submission system

Fair and swift peer-review system

Efficient typesetting and proofreading procedure

Display of the result of downloads and visits, as well as the number of cited articles

Maximum dissemination of your research work

Submit your manuscript at: http://papersubmission.scirp.org/

Or contact ojpsych@scirp.org 